\title{
Comparación de dos funcionales de optimización para el control de estructuras monosimétricas sometidas a sismos de ancho de banda amplio por medio de dos amortiguadores de columna de líquido sintonizados
}

\author{
Comparison of two optimization functionals for the control of monosymmetric structures \\ subject to broad bandwidth earthquakes by means of two tuned liquid column dampers
}

Fecha de entrega: 27 de marzo 2021

Fecha de aceptación 22 de octubre 2021

\section{Gilda Espinoza, Euro Casanova, Nicole Moya, Gerardo Coloma y Álvaro Suazo}

Departamento de Ingeniería Civil y Ambiental, Universidad del Bío-Bío, Avenida Collao 1202, Casilla 5-C, Concepción, Chile, gespinoz@ubiobio.cl,ecasanova@ubiobio.cl,nimoya@egresados.ubiobio.cl,gecoloma@egresados.ubiobio.cl,asuazo@ubiobio.cl

En este trabajo se estudia el comportamiento de dos amortiguadores de columna de líquido sintonizados (ACLS), en el control torsional de una estructura asimétrica de comportamiento lineal, ante una excitación sísmica de alto contenido de frecuencias. El modelo de estudio es una estructura monosimétrica en dirección $X$ a la que se le han adicionado dos ACLS en dirección Y, para controlar el movimiento de la estructura con acoplamiento lateral-torsional. Se consideran dos criterios de optimización, cada uno representando una forma alternativa de determinar la demanda de deformación de los bordes de la planta. El primer criterio consiste en igualar las varianzas de deformación de los bordes de la planta. El segundo criterio consiste en igualar las desviaciones estándar de deformación de los bordes de la planta. El objetivo final es balancear torsionalmente la estructura. Los resultados muestran un balance torsional prácticamente idéntico, con los ACLS optimizados con cada uno de los criterios, a excepción del caso de una razón de frecuencia torsional flexible y una razón de masa baja. Además, se encuentra que la posición óptima de los ACLS para excentricidades mayores a 0.12, sigue la misma regla de comportamiento de la posición óptima de los amortiguadores de masa sintonizados.

Palabras clave: amortiguador de columna de líquido sintonizado, optimización, balance torsional, análisis estocástico
This work focuses on the study of two tuned liquid column dampers (TLCD) for the torsional control of an asymmetric structure with linear behaviour, subjected to a seismic excitation of high frequency content. The model under study is a X-direction monosymmetric structure with two Y-direction TLCD to control the movement of the structure with lateraltorsional coupling. Two optimization criteria are considered, each one representing an alternative way of defining the deformation demand of the plant edges. The first criterion consists of equalizing the deformation variances of the plant edges. The second criterion involves matching the standard deviations of deformation of the edges of the plant. The final objective is to torsionally balance the structure. The results show a practically identical torsional balance for the two used criteria, except in the case of a flexible torsional frequency ratio and a low mass ratio. Furthermore, it is found that the optimal position of the TLCD for eccentricities greater than 0.12 follows the same behavior rule of the optimal position of the tuned mass dampers.

Keywords: tuned liquid column damper, optimization, torsional balance, stochastic analysis

\section{Introducción}

Durante los últimos años han surgido diversos sistemas de protección sísmica, como una forma de diseño sismorresistente alternativo al diseño tradicional. Este diseño se basa en disminuir la demanda sobre la estructura, producida por una excitación sísmica, a través de la inclusión de dispositivos de reducción de vibraciones. Estos sistemas reducen alguna respuesta de interés, 
como por ejemplo el desplazamiento que experimentará la estructura, disminuyendo así la demanda sobre ésta. Dentro de estos dispositivos se encuentran los sistemas efecto-masa, entre ellos se encuentran el amortiguador de masa sintonizados (AMS) y el amortiguador de columna de líquido sintonizado (ACLS). Ambos se basan en el mismo principio, sintonizar la frecuencia del dispositivo con la frecuencia fundamental de la estructura y así disminuir los desplazamientos de la estructura principal.

El dispositivo ACLS es el estudiado en esta investigación. El ACLS fue propuesto por Sakai et al. (1989) con el fin de reducir los desplazamientos en estructuras sometidas a viento. El dispositivo consiste en un tubo en $U$ en el que un líquido puede oscilar libremente, lo que hace a una frecuencia particular que depende de los parámetros físicos del dispositivo (frecuencia propia). Además, posee un orificio en su sección horizontal (parte inferior del tubo) que aporta amortiguamiento al sistema a través de la pérdida de carga producida por la restricción.

Las investigaciones posteriores sobre este tipo de dispositivo se enfocaron en el estudio del comportamiento del ACLS en el control de estructuras ante excitaciones sísmicas. Dentro de estos estudios, se encuentra el de Won et al. (1996), quienes concluyeron que los parámetros óptimos de diseño del ACLS (razón de sintonía entre la frecuencia el dispositivo y la frecuencia lateral desacoplada de la estructura, el coeficiente de pérdida de carga y la razón entre la masa del líquido contenido en el dispositivo y la masa del sistema principal), dependen del contenido de frecuencia, intensidad y duración de la excitación sísmica.

Yalla y Kareem (2000), analizaron el comportamiento del ACLS a través de un análisis estocástico, considerando cargas de viento y sismo, representadas a través de ruido blanco y ruido blanco filtrado, usando una estructura de un grado de libertad no amortiguada. Consideraron una linealización estadística equivalente para el ACLS y propusieron una solución explícita para el coeficiente de pérdida de carga y razón de sintonía óptima para el ACLS. Shum (2009) desarrolló un esquema de solución de forma cerrada para los parámetros óptimos de diseño de un ACLS en una estructura no amortiguada, validando su propuesta mediante comparación contra el método iterativo convencional. Chakraborty et al. (2012) estudiaron el efecto de la restricción que presenta el movimiento máximo del flujo en el ACLS en los parámetros óptimos de éste, en estructuras sometidas a excitaciones sísmicas.

Otros estudios se han orientado a modificar el diseño del ACLS con el objetivo de obtener mejores desempeños. Dentro de estos estudios se encuentra el realizado por Rozas et al. (2016), quienes propusieron un ACLS bidireccional, es decir, dos ACLS orientados perpendicularmente el uno respecto al otro. Los autores concluyen que este tipo de configuración puede disminuir la respuesta ante eventos sísmicos bidireccionales, requiriendo una menor masa de agua con respecto a dos ACLS equivalentes.

Un aspecto relevante en el diseño de edificios es la existencia de excentricidad debida a diversas causas (consideraciones arquitectónicas). Esto se traduce en estructuras asimétricas, en las que el centro de masa se encuentra a una distancia (excentricidad) del centro de rigidez. Esta asimetría en rigidez provoca un fenómeno denominado torsión, produciéndose desplazamientos de giro en la planta. Este comportamiento es muy dañino en una estructura, por lo tanto, no es admisible bajo las normativas vigentes, debido a que puede provocar daño estructural y no estructural. Los primeros estudios sobre la eficiencia del dispositivo ACLS en el control torsional fueron realizados por Shum y Xu (2002), quienes investigaron el rendimiento de múltiples ACLS (MACLS) en la reducción de la respuesta torsional de estructuras, en comparación con un solo ACLS, encontrando que, para una misma razón de masa, los MACLS tienen un mejor desempeño con respecto a un solo ACLS.

Un punto muy importante al obtener los parámetros de diseño es el criterio utilizado en la optimización de los dispositivos. Sgobba y Marano (2010) obtuvieron los parámetros óptimos de diseño de un AMS para la protección de estructuras no lineales sometidas a sismos, a través de la minimización de tres funcionales. El primero, considera la minimización del desplazamiento del sistema principal, el segundo consiste en la minimización de la energía histerética disipada por la estructura, y el tercero consiste en la minimización de un ponderado de ambos criterios. Estos autores concluyeron que para el caso estudiado no existen grandes diferencias en los parámetros encontrados para los tres funcionales. Por otro lado, Espinoza et al. (2018a), analizaron el comportamiento de los parámetros óptimos de un ACLS en el control de una estructura no 
lineal empleando los dos primeros criterios de Sgobba y Marano (2010), concluyendo que el comportamiento de los parámetros óptimos del ACLS, para ambos criterios es muy similar, al igual que lo encontrado con el AMS.

En estructuras asimétricas, de la Llera et al. (2005) plantearon un criterio de control torsional, llamado balance torsional (BT) para estructuras sometidas a excitaciones sísmicas. El BT consiste en igualar las demandas de deformación de los bordes de la estructura para así reducir el daño provocado en la estructura, debido al giro y traslación desacopladas de la planta. Este criterio ha sido utilizado en la optimización de distintos dispositivos para el control de estructuras asimétricas como disipadores friccionales (de la Llera et al., 2005), disipadores viscoelásticos (García et al., 2007), disipadores viscosos (Almazán y de la Llera, 2009), AMS (Almazán et al., 2012, Espinoza et al., 2019) y los sistemas de aislación basal (Seguín et al., 2013). En cuanto a los criterios de optimización usados en el caso de BT, Espinoza et al (2018b) propusieron dos criterios de optimización para el AMS en el control de estructuras no lineales con comportamiento lateral torsional. El primer criterio consiste en el balance uniforme y reducción de la energía histerética, simultáneamente en los planos no lineales. El segundo se basa en la minimización del daño de la estructura basado en la media entre la energía histerética normalizada, y el coeficiente de correlación entre el desplazamiento y rotación de la planta.

Las investigaciones descritas en los párrafos anteriores estudian la utilización de distintos funcionales para el control de estructuras principalmente simétricas. Sin embargo, en estructuras asimétricas, en las que existe un movimiento lateral torsional, en el ámbito estocástico sólo se ha utilizado el criterio de BT (de la Llera et al., 2005) y aplicado en la optimización de AMS. Por lo tanto, es importante analizar nuevos funcionales para el control de distintos dispositivos de control de vibraciones ante excitaciones sísmicas.

El objetivo de la presente investigación es analizar el comportamiento de dos ACLS óptimos, en el control de una estructura asimétrica, sometida a una excitación sísmica estocástica. El input sísmico, se considerará de ancho de banda amplio. Se utilizarán dos funcionales, el primero es la forma clásica del criterio de BT y el segundo es una variante del criterio de BT.

\section{Modelo estructural}

Esta investigación considera un sistema estructural monosimétrico de un piso, en lo siguiente denominado Modelo $1\left(\mathrm{M}_{1}\right)$, que se muestra en la Figura 1. El sistema $M_{1}$ tiene excentricidad en dirección $X$ y una relación de largo-ancho igual a $4(a / b=4)$. La masa traslacional de $\mathrm{M}_{1}$ es $m_{s}$, el radio de giro de la planta es $p_{s}$, y el centro geométrico (CG) coincide con el centro de masa (CM). El centro de rigidez $(\mathrm{CR})$ está a una distancia $e_{s x}$ del CM. El modelo $\mathrm{M}_{1}$ posee un período lateral $T_{s y}$, asociado al modo donde predomina la traslación en $\mathrm{Y}$, y un período torsional $T_{s \theta}$, asociado al modo donde predomina la torsión. Por otra parte, $\Omega=T_{s y} / T_{s \theta}$ es la relación de frecuencia torsional para el modelo $\mathrm{M}_{1}$. Se considera una razón de amortiguamiento $\xi_{s}=0.002$ en todos los modos de vibración de la estructura principal.

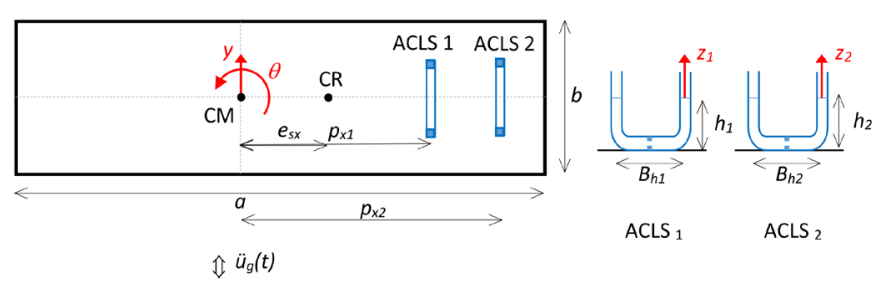

Figura 1: Modelo $1\left(\mathrm{M}_{1}\right)$

\section{Ecuaciones del movimiento}

A continuación, se presenta la teoría en la que se basa la formulación de las ecuaciones del movimiento del sistema $\mathrm{M}_{1}$.

\section{Ecuaciones del movimiento del ACLS}

La ecuación del movimiento de un ACLS colocado sobre una estructura de un grado de libertad, sometida a una aceleración basal $\ddot{u}_{g}$ (Sakai et al., 1989), se expresa bajo la forma:

$\rho_{l} A L_{e} \ddot{z}+\frac{1}{2} \rho_{l} A \xi_{l}|\dot{z}| \dot{z}+2 \rho_{l} g A z=-\rho_{l} A B_{h}\left(\ddot{x}+\ddot{u}_{g}\right)$

Donde $\ddot{x}$ representa la aceleración de la estructura, $z$ describe el movimiento del líquido al interior del ACLS, $\rho_{l}$ es la densidad de líquido, $A$ es el área de la sección transversal del tubo, $L_{e}=2 h+B_{h}$ es la longitud total de la columna líquida formada por la longitud horizontal $B_{h}$ y la longitud vertical $h ; g$ es la aceleración de gravedad y $\xi_{l}$ es el coeficiente de pérdida de carga de la singularidad 
producida por el orificio en el parte inferior del ACLS.

Para hacer un análisis estocástico se realiza una linealización estadística equivalente del término no lineal de la ecuación (1), lo que se muestra en la ecuación (2). Considerando un proceso estacionario Gaussiano de media cero, se tiene el coeficiente de amortiguamiento lineal equivalente $c_{p}$, que se muestra en la ecuación (3) (Roberts y Spanos, 2003).

$$
\begin{aligned}
& \rho_{l} A L_{e} \ddot{z}+2 \rho_{l} A c_{p} \dot{z}+2 \rho_{l} g A z=-\rho_{l} A B_{h}\left(\ddot{x}+\ddot{u}_{g}\right) \\
& c_{p}=\frac{\xi \sigma_{\dot{z}}}{\sqrt{2 \pi}}
\end{aligned}
$$

donde $\sigma_{z}$ es la desviación estándar de la velocidad del líquido del ACLS.

Normalizando (2) respecto a la masa del líquido, se obtiene:

$$
\ddot{z}+\frac{2 c_{p}}{L_{e}} \dot{z}+\frac{2 g}{L_{e}} z+p \ddot{x}=-p \ddot{u}_{g}
$$

donde $p=B_{h} / L_{e}$ es la razón de longitud del ACLS. A partir de (4) se puede definir la frecuencia del líquido como $\omega_{l}=\sqrt{2 g / L_{e}}$. Denotando la frecuencia desacoplada en dirección $\mathrm{Y}$ del modelo $\mathrm{M}_{1}$ como $\omega_{s y}=2 \pi / T_{s y}$, entonces se puede definir $\gamma_{l}=\omega_{l} / \omega_{s y}$ como la razón de sintonía del ACLS con respecto a la frecuencia desacoplada del sistema principal.

\section{Ecuaciones del movimiento de $\mathrm{M}_{1}$}

A continuación, se derivan las ecuaciones del movimiento del modelo $\mathrm{M}_{1}$. Para ello, se define el vector de grados de libertad del modelo como:

$$
\mathbf{X}(\mathrm{t})=\left[\begin{array}{llll}
y(t) & \theta(t) & z_{1}(t) & z_{2}(t)
\end{array}\right]^{T}
$$

donde $y(t)$ representa el desplazamiento traslacional en dirección $\mathrm{Y}, \theta(t)$ describe el ángulo de rotación de la planta y $z_{1}(t)$ y $z_{2}(t)$ describen el desplazamiento del líquido al interior de los ACLS y $\mathrm{ACLS}_{2}$, respectivamente. Así, la ecuación de equilibrio dinámico del modelo $\mathrm{M}_{1}$ se escribe de la siguiente forma:

$$
\mathbf{M} \ddot{\mathbf{X}}+\mathbf{C} \dot{\mathbf{X}}+\mathbf{K X}=-\mathbf{B}_{\mathbf{u}} \ddot{u}_{g}
$$

donde $\mathbf{M}, \mathbf{C}$ y $\mathbf{K}$, y son las matrices de masa (ecuación
(7)), amortiguamiento (8) y rigidez (9), respectivamente. Además, $\mathbf{B}_{u}$ es la matriz de influencia del input (ecuación (10)) y $\ddot{u}_{g}$ es la aceleración del suelo en la dirección Y.

$\mathbf{M}=\left[\begin{array}{cccc}1+\mu_{l 1}+\mu_{l 2} & \mu_{l 1} p_{x 1}+\mu_{l 2} p_{x 2} & \mu_{l 1} p_{1} & \mu_{l 2} p_{2} \\ \mu_{l 1} p_{x 1}+\mu_{l 2} p_{x 2} & \rho_{s}^{2}+\mu_{l 1} p_{x 1}^{2}+\mu_{l 2} p_{x 2}^{2} & \mu_{l 1} p_{x 1} p_{1} & \mu_{l 2} p_{x 2} p_{2} \\ \mu_{l 1} p_{1} & \mu_{l 1} p_{x 1} p_{1} & \mu_{l 1} & 0 \\ \mu_{l 2} p_{2} & \mu_{l 2} p_{x 2} p_{2} & 0 & \mu_{l 2}\end{array}\right]$

$\mathbf{C}=\left[\begin{array}{cccc}2 \xi_{s} \omega_{s y} & 2 \xi_{s} \omega_{s y} e_{s x} & 0 & 0 \\ 2 \xi_{s} \omega_{s y} e_{s x} & C_{r} / M_{s}+2 \xi_{s} \omega_{s y} e_{s x}^{2}+\mu_{l 1} p_{x 1}^{2}+\mu_{l 2} p_{x 2}^{2} & 0 & 0 \\ 0 & 0 & 2 \frac{C p_{1}}{L_{e 1}} \mu_{l 1} & 2 \frac{C p_{2}}{L_{e 2}} \mu_{l 2}\end{array}\right]$

$\mathbf{K}=\left[\begin{array}{cccc}\omega_{s y}^{2} & \omega_{s y}^{2} e_{s x} & 0 & 0 \\ \omega_{s y}^{2} e_{s x} & \omega_{s y}^{2}\left(\Omega_{\theta}^{2} \rho_{s}^{2}+e_{s x}^{2}\right) & 0 & 0 \\ 0 & 0 & \frac{g}{L_{e 1}} \mu_{l 1} & 0 \\ 0 & 0 & 0 & 2 \frac{g}{L_{e 2}} \mu_{l 2}\end{array}\right]$

$\mathbf{B}_{\mathbf{u}}=\left[\begin{array}{c}1+\mu_{l 1}+\mu_{l 2} \\ \mu_{l 1} p_{x 1}+\mu_{l 2} p_{x 2} \\ \mu_{l 1} p_{1} \\ \mu_{l 2} p_{2}\end{array}\right]$

En estas expresiones $\mu_{l 1}$ y $\mu_{l 2}$ representan las razones de masa del líquido contenido en el $\mathrm{ACLS}_{1}$ y en el $\mathrm{ACLS}_{2}$, respectivamente.

\section{Excitación estocástica}

Con el fin de realizar el estudio sísmico estocástico, se considera una función de la densidad de potencial espectral (DPE) representativa con una excitación sísmica de ancho de banda amplio (BBP). Para obtener el ancho de banda deseado, se ajustó un filtro de Clough-Penzien (FCP) (Clough y Penzien, 1975), a la DPE de un sismo artificial compatible con el espectro de la norma chilena NCh2745 (2013) para suelo tipo B. Este espectro es representativo de sismos de alto contenido de frecuencias, típicos de Chile.

La ecuación del filtro utilizado en este estudio es:

$S_{g}(\omega)=S_{0} \frac{\omega_{g}^{4}+4 \xi_{g}^{2} \omega_{g}^{2} \omega^{2}}{\left(\omega_{g}^{2}-\omega^{2}\right)^{2}+4 \xi_{g}^{2} \omega_{g}^{2} \omega^{2}}$

$\frac{\omega^{4}}{\left(\omega_{f}^{2}-\omega^{2}\right)^{2}+4 \xi_{f}^{2} \omega_{f}^{2} \omega^{2}}$ 
donde los parámetros ajustados a la DPE, a través de mínimos cuadrados, se muestran en la Tabla 1.

Tabla 1: Parámetros del filtro Clough - Penzien

\begin{tabular}{|c|c|c|c|c|c|}
\hline \multirow{2}{*}{ Tipo de entrada } & \multicolumn{5}{|c|}{ Parámetros } \\
\cline { 2 - 6 } & $S_{0}$ & $\omega_{g}, \mathrm{rad} / \mathrm{s}$ & $\xi_{g}$ & $\omega_{f}, \mathrm{rad} / \mathrm{s}$ & $\xi_{f}$ \\
\hline BBP & 1335.6 & 3.05 & 0.041 & 8.48 & 0.90 \\
\hline
\end{tabular}

\section{Formulación espacio-estado}

Con el fin de incorporar la excitación sísmica estocástica al sistema $\mathrm{M}_{1}$ se expresan las ecuaciones de movimiento en formulación espacio-estado. Definiendo el vector de estado como:

$$
\mathbf{X}_{1}=\left[\begin{array}{ll}
\mathbf{X} & \dot{\mathbf{X}}
\end{array}\right]^{\mathrm{T}}
$$

La ecuación (6) toma la forma:

$$
\dot{\mathbf{X}}_{1}=\mathbf{A} \mathbf{X}_{1}+\mathbf{B} \ddot{u}_{g}
$$

donde A es la matriz de estado (ecuación (14)) y $\mathbf{B}$ es el vector de colocación del input (ecuación (15)).

$$
\begin{aligned}
& \mathbf{A}=\left[\begin{array}{cc}
\mathbf{0}_{4 \times 4} & \mathbf{I}_{4 \times 4} \\
-\mathbf{M}^{-1} \mathbf{K} & -\mathbf{M}^{-1} \mathbf{C}
\end{array}\right] \\
& \mathbf{B}=\left\{\begin{array}{c}
\mathbf{0}_{4 \times 1} \\
-\mathbf{M}^{-1} \mathbf{B}_{\mathbf{u}}
\end{array}\right\}
\end{aligned}
$$

Por otra parte, las ecuaciones en la formulación espacioestado del filtro se obtienen definiendo el vector de estado del filtro como:

$$
\mathbf{X}_{\mathbf{f}}=\left[\begin{array}{llll}
x_{g} & \dot{x}_{g} & x_{f} & \dot{x}_{f}
\end{array}\right]^{\mathrm{T}}
$$

Así, las ecuaciones del filtro toman la forma:

$$
\dot{\mathbf{X}}_{\mathrm{f}}=\mathbf{A}_{\mathbf{f}} \mathbf{X}_{\mathbf{f}}+\mathbf{B}_{\mathrm{f}} \mathrm{W}(\mathrm{t})
$$

donde $\mathbf{A}_{\mathbf{f}}$ (ecuación (18)) es la matriz de estado del filtro, $\mathbf{B}_{\mathbf{f}}$ es el vector de colocación del input (19) y es la intensidad del ruido blanco en el tiempo.

$$
\mathbf{A}_{\mathbf{f}}=\left[\begin{array}{cccc}
0 & 1 & 0 & 0 \\
-\omega_{g}^{2} & -2 \xi_{g} \omega_{g} & 0 & 0 \\
0 & 0 & 0 & 1 \\
\omega_{g}^{2} & 2 \xi_{g} \omega_{g} & -\omega_{f}^{2} & -2 \xi_{f} \omega_{f}
\end{array}\right]
$$

$$
\mathbf{B}_{\mathbf{f}}=\left[\begin{array}{c}
0 \\
-1 \\
0 \\
0
\end{array}\right]
$$

Finalmente, para incluir la excitación sísmica estocástica en el modelo $\mathrm{M}_{1}$, se define el vector de espacio-estado global $\mathbf{X}_{2}$ :

$$
\mathbf{X}_{2}=\left[\begin{array}{ll}
\mathbf{X}_{1} & \mathbf{X}_{\mathrm{f}}
\end{array}\right]^{\mathrm{T}}
$$

obteniendo la ecuación total del sistema:

$$
\dot{\mathbf{X}}_{2}=\mathbf{A}_{2} \mathbf{X}_{2}+\mathbf{B}_{2} \mathrm{~W}(\mathrm{t})
$$

donde:

$$
\begin{aligned}
& \mathbf{A}_{2}=\left[\begin{array}{cc}
\mathbf{A} & \mathbf{B ~ C}_{\mathrm{f}} \\
\mathbf{0}_{4 \times 4} & \mathbf{A}_{\mathrm{f}}
\end{array}\right] \\
& \mathbf{B}_{2}=\left\{\begin{array}{c}
\mathbf{0}_{8 \times 1} \\
\mathbf{B}_{\mathrm{f}}
\end{array}\right\}
\end{aligned}
$$

Con $\mathbf{C}_{\mathrm{f}}$ el vector fila de output del filtro definido como (Saitua et al., 2018):

$$
\mathbf{C}_{\mathbf{f}}=\left[\begin{array}{llll}
\omega_{g}^{2} & 2 \xi_{g} \omega_{g} & -\omega_{f}^{2} & -2 \xi_{f} \omega_{f}
\end{array}\right]
$$

\section{Obtención de la matriz de covarianza}

Con el fin de estudiar el comportamiento del sistema ante una excitación estocástica, se asumió un estado estacionario considerando como input un ruido blanco. Para la definición de la potencia del ruido blanco, se consideró que la varianza de aceleración del suelo a la entrada de la estructura es:

$$
\sigma_{\ddot{u}_{g}}^{2}=\frac{P G A}{3}
$$

donde PGA denota la aceleración máxima del terreno (Peak Ground Aceleration). En este estudio se consideró un PGA de $0.3 \mathrm{~g}$, donde g es la aceleración de gravedad. Con el fin de obtener la matriz de covarianza del sistema analizado se aplicó la ecuación de Lyapunov a la ecuación (21), obteniendo: 
$\boldsymbol{A}_{\mathbf{2}}^{T} \boldsymbol{R}_{\mathbf{1}}+\boldsymbol{R}_{\mathbf{1}} \boldsymbol{A}_{\mathbf{2}}+\boldsymbol{B}_{2}^{T} W B_{\mathbf{2}}=0$

donde $\mathbf{R}_{\mathbf{1}}$ es la matriz de covarianza del sistema completo estructura-ACLS-filtro, excitado por un ruido blanco.

\section{Procedimiento de optimización}

A continuación, se presentan los criterios de optimización considerados en este estudio. Para ello se determinará el vector de covarianza de deformación de los bordes $\mathbf{V}_{\mathrm{b}}$, según $\mathrm{Y}$, a través de la siguiente expresión:

$$
\mathbf{V}_{\mathbf{b}}=\operatorname{diag}\left(\mathbf{L}_{\mathbf{b}} \boldsymbol{V}_{\boldsymbol{q}} \mathbf{L}_{\mathbf{b}}^{\mathbf{T}}\right)
$$

donde $\mathbf{V}_{q}$ es la sub-matriz de la matriz covarianza $\mathbf{R}_{\mathbf{1}}$ correspondiente a los grados de libertad de traslación y rotación de la estructura $(y(t)$ y $\theta(t))$, y $\mathbf{L}_{\mathbf{b}}$ es la matriz de transformación cinemática entre las deformaciones de borde y los grados de libertad.

$$
\mathbf{L}_{\mathbf{b}}=\left[\begin{array}{cc}
1 & -a / 2 \\
1 & a / 2
\end{array}\right]
$$

\section{Criterio 1: Balance torsional}

En este trabajo, el criterio 1 para optimización de los ACLS está basado en el criterio de BT propuesto por de la Llera et al. (2005), el cual establece que para que se produzca un BT se deben igualar las demandas de deformación de los bordes de la planta, lo que se traduce en igualar las varianzas de desplazamientos de los bordes de la planta. La ecuación (29) muestra dicho criterio aplicado a la optimización de los ACLS.

Minimizar:

$$
\begin{aligned}
& J_{1}\left(\gamma_{l 1}, \gamma_{l 2}, \xi_{l 1}, \xi_{l 2}, p_{x 1}, p_{x 2}\right)=\max \left(\mathbf{V}_{\mathbf{b}}\right) \\
& \text { Sujeto a: }\left\{\begin{array}{c}
0.5<\gamma_{l 1}<1.5 \\
0.5<\gamma_{l 2}<1.5 \\
\xi_{l 1}>0 \\
\xi_{l 2}>0 \\
-\frac{a}{2}<p_{x 1}<\frac{a}{2} \\
-\frac{a}{2}<p_{x 2}<\frac{a}{2} \\
h_{1}-c \sigma_{z 1} \geq 0 \\
h_{2}-c \sigma_{z 2} \geq 0
\end{array}\right.
\end{aligned}
$$

donde $\gamma_{l 1} \mathrm{y} \gamma_{l 2}, \xi_{l 1}$ y $\xi_{l 2}, p_{\mathrm{x} 1}$ y $p_{\mathrm{x} 2}, h_{1}$ y $h_{2}$ son las frecuencias, los coeficientes de pérdida de carga, las posiciones en dirección X y las alturas de las columnas de agua, en dirección $\mathrm{Z}$ y las razones de sintonía del ACLS 1 y ACLS , $_{2}$ respectivamente. $\xi_{l 1}$ y $\xi_{l 2}$ son los coeficientes de pérdida de carga del $\mathrm{ACLS}_{1}$ y $\mathrm{ACLS}_{2}, p_{\mathrm{x} 1}$ y $p_{\mathrm{x} 2}$ es la posición del $\mathrm{ACLS}_{1}$ y $\mathrm{ACLS}_{2}$ en dirección $\mathrm{X}, h_{1}$ y $h_{2}$ son las alturas de las columnas de agua en dirección Z, del ACLS 1 y ACLS respectivamente.

\section{Criterio 2: Variación del criterio de balance torsional}

El criterio 2 considerado en este trabajo es una variación del BT, donde en lugar de igualar las varianzas de desplazamientos de los bordes de la planta, se igualan las desviaciones estándar de los bordes de la planta, manteniendo las mismas restricciones usadas en el criterio 1.

Minimizar: $J_{1}\left(\gamma_{l 1}, \gamma_{l 2}, \xi_{l 1}, \xi_{l 2}, p_{x 1}, p_{x 2}\right)=\max \left(\mathbf{v}_{\mathbf{b}}{ }^{1 / 2}\right)$

\section{Resultados y discusión}

A continuación, se realiza un análisis de sensibilidad de los parámetros óptimos, con respecto a una variación de la razón de excentricidad normalizada y a la razón de masa del líquido. Posteriormente, se comprueba el balance torsional para los dos criterios.

\section{Análisis del comportamiento de los parámetros óptimos}

Las Figuras 2, 3, 4, 5, 6 y 7 muestran el comportamiento de los parámetros óptimos, de cada uno de los ACLS. En las Figuras 2, 3, 5 y 6, los parámetros óptimos se presentan en función de la excentricidad normalizada respecto al radio de giro de la planta y la razón de masa del líquido total. Esta última es la suma del líquido de ambos ACLS, con respecto a la masa del sistema principal. Las Figuras 4 y 7 sólo consideran el caso de $\mu_{l}=0.06$ y $\mu_{l}=0.08$, para todo el rango excentricidades normalizadas. Todas las figuras fueron generadas para una razón de longitud de los ACLS de 0.5 para cada ACLS, considerando que la masa de líquido para cada ACLS era un $50 \%$ de la masa de líquido total y que la razón de amortiguamiento para la estructura principal era de $2 \%$. Además, se consideró un período de $2 \mathrm{~s}$ para la estructura principal. En los gráficos de cada figura, se muestran los dos criterios de optimización utilizados. El análisis se realiza primero para una estructura torsionalmente flexible $\Omega=0.7$, y posteriormente para una estructura torsionalmente rígida $\Omega=1.3$. 
Espinoza, G., Casanova, E., Moya, N., Coloma, G. y Suazo, A. (2021). Comparación de dos

funcionales de optimización para el control de estructuras monosimétricas sometidas a sismos de ancho de banda amplio por medio de dos amortiguadores de columna de líquido sintonizados Obras y Proyectos 30, 50-59

La Figura 2 muestra la razón de frecuencias óptima para los dos ACLS del modelo $\mathrm{M}_{1}$ para el caso de una estructura torsionalmente flexible. Las Figuras $2 \mathrm{a}$ y $2 \mathrm{~b}$ corresponden al $\mathrm{ACLS}_{1}$ y ACLS $_{2}$, respectivamente. En ambas gráficas se muestran las superficies para los dos criterios estudiados.

a)

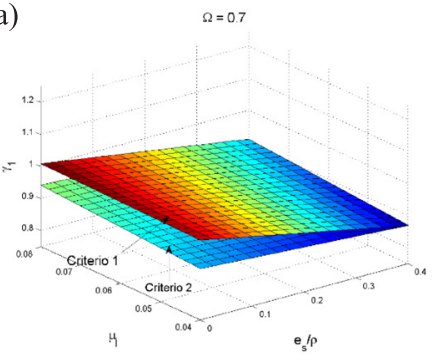

Figura 2: Razón de frecuencias óptimas, criterios 1 y $2, p_{1}=p_{2}=$ $0.5, \xi_{s}=0.02, \Omega=0.7:$ a) $\operatorname{ACLS}_{1}$ y b) $\operatorname{ACLS}_{2}$

En las Figuras 2a y $2 \mathrm{~b}$ se observa que la razón de frecuencias óptima, en cada ACLS, es insensible a la razón de masa del líquido, sin embargo, disminuye a medida que aumenta la excentricidad normalizada de la planta. Por otra parte, el comportamiento del valor de la razón de frecuencias óptima de ambos ACLS es prácticamente el mismo. También se observa que la razón de frecuencias óptima del $\mathrm{ACLS}_{2}$ obtenida a través del criterio 1, es mayor a la razón de frecuencia óptima obtenida con el criterio 2.

a)
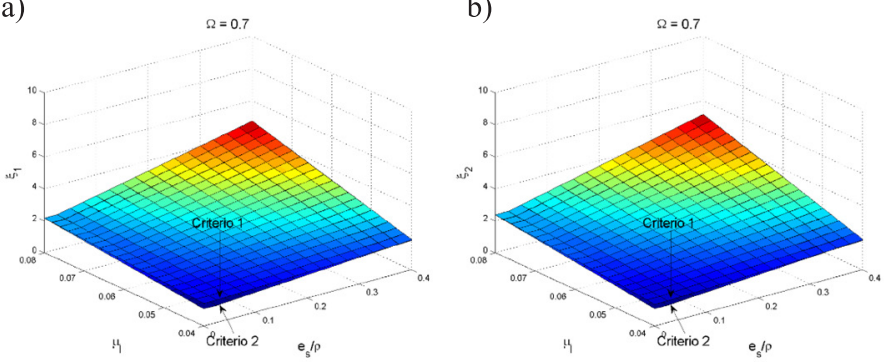

Figura 3: Coeficientes de pérdida de carga óptimos, criterio 1 y $2, p_{1}=p_{2}=0.5, \xi_{s}=0.02, \Omega=0.7:$ a) $\operatorname{ACLS}_{1}$ y b) $\mathrm{ACLS}_{2}$

La Figura 3 muestra el comportamiento del coeficiente de pérdida carga óptimo para cada ACLS para una estructura torsionalmente flexible. Allí se observa que el valor de este parámetro óptimo prácticamente no depende ni del criterio de optimización empleado, ni del ACLS estudiado. Sin embargo, se observa un aumento del valor del coeficiente de pérdida de carga óptimo, a medida que aumenta la razón de masa y la excentricidad normalizada.

La Figura 4 muestra el comportamiento de la posición óptima normalizada por el largo de la planta para cada ACLS para una estructura torsionalmente flexible. La
Figura 4a para $\mathrm{ACLS}_{1}$ (fila superior) y la Figura $4 \mathrm{~b}$ para $\mathrm{ACLS}_{2}$ (fila inferior). La primera columna considera una razón de masa $\mu_{1}=0.06$ y la segunda $\mu_{1}=0.08$. En la Figura 4 se observa que cuando la razón de masa es mayor, el comportamiento de la posición óptima es el mismo independiente del criterio de optimización. Ambos ACLS se ubican en la misma posición, es decir, en el borde más cercano al CR (borde rígido), por lo tanto, no es necesario considerar dos ACLS, basta con uno. Este resultado es coincidente con los encontrados para un AMS por Almazán et al. (2012). Sin embargo, cuando la razón de masa es menor, el criterio considerado sí toma relevancia, ya que el ACLS $_{1}$ se ubica en el borde opuesto.
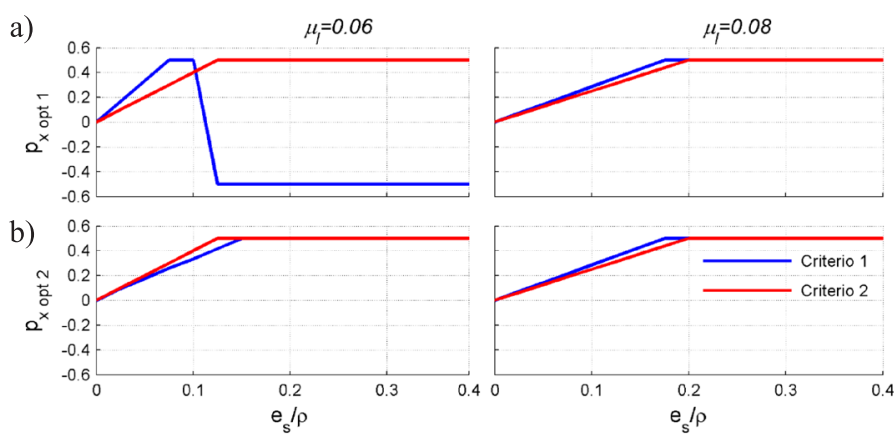

Figura 4: Posiciones óptimas, criterios 1 y $2, p_{1}=p_{2}=0.5$, $\xi_{s}=$ $0.02, \Omega=0.7$ : a) $\operatorname{ACLS}_{1}$ y b) $\operatorname{ACLS}_{2}$

Las Figuras 5 a 7 muestran resultados análogos a los mostrados en las Figuras 2 a 4, pero considerando una razón de frecuencia torsional $\Omega=1.3$, es decir, para una estructura torsionalmente rígida. Así, la Figura 5 presenta el comportamiento de la razón de frecuencias óptima, observándose el mismo comportamiento de la razón de frecuencias óptima para ambos ACLS y ambos criterios, en función del valor de la razón de masa y excentricidad normalizada. Por otra parte, se observa un cambio en la magnitud de la razón de frecuencias óptima, dependiendo del criterio considerado para cada ACLS.
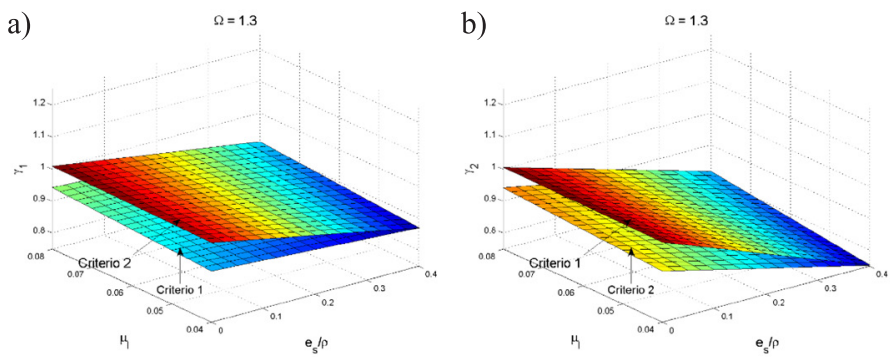

Figura 5: Razón de frecuencias óptimas, criterios 1 y $2, p_{1}=p_{2}=$ $0.5, \xi_{s}=0.02, \Omega=1.3$ : a) $\operatorname{ACLS}_{1}$ y b) $\mathrm{ACLS}_{2}$ 
La Figura 6 muestra el coeficiente de pérdida de carga óptimo para cada ACLS, en el caso de una estructura torsionalmente rígida. Los resultados muestran un comportamiento prácticamente idéntico al obtenido para $\Omega=0.7$, es decir, el valor del coeficiente de pérdida de carga es insensible al criterio de optimización utilizado.
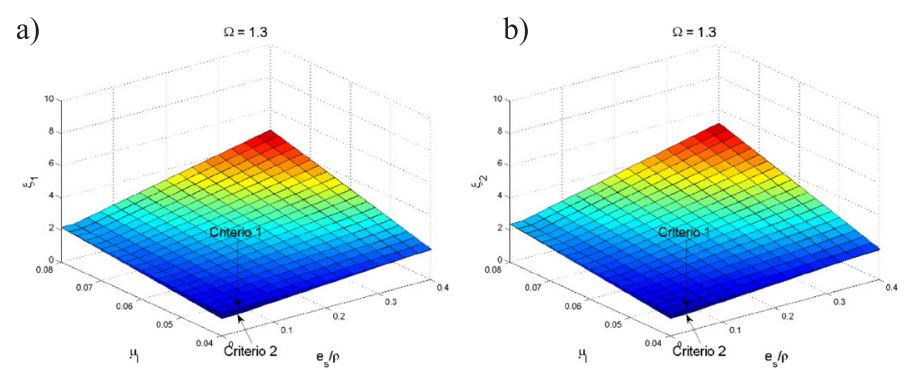

Figura 6: Coeficiente de pérdida de carga óptimos, criterios 1 y $2, p_{1}=p_{2}=0.5, \xi_{s}=0.02, \Omega=1.3:$ a) $\operatorname{ACLS}_{1}$ y b) $\operatorname{ACLS}_{2}$

La Figura 7 muestra el comportamiento de la posición óptima en planta de cada ACLS para una estructura torsionalmente rígida. En la Figura $7 \mathrm{a}$ se observa que el comportamiento de este parámetro óptimo es independiente del criterio utilizado para el $\mathrm{ACLS}_{1}$, pero sí es dependiente en el caso del ACLS $_{2}$ (Figura 7b). Además, se observa que existe un cambio en la posición de ambos ACLS dependiendo de la excentricidad. La posición óptima en ambos ACLS coincide con el comportamiento de este parámetro óptimo para un AMS, cuando la razón de excentricidad normalizada es mayor a 0.12 . En este caso la posición óptima se ubica en el borde flexible (borde más alejado del CR), cuando la estructura es torsionalmente rígida (Almazán et al., 2012).

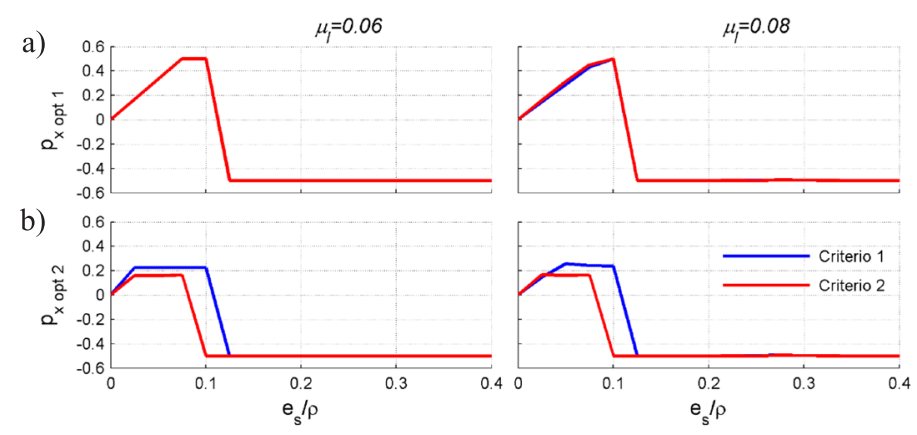

Figura 7: Posiciones óptimas, criterios 1 y 2, $p_{1}=p_{2}=0.5$, $\xi_{s}=$ $0.02, \Omega=1.3:$ a) $\operatorname{ACLS}_{1}$ y b) $\operatorname{ACLS}_{2}$

\section{Análisis del cumplimiento del balance torsional}

La Figura 8 muestra el cumplimiento del balance torsional al utilizarse el criterio en función de la varianza del desplazamiento de los bordes (criterio 1) o el criterio en función de la desviación estándar del desplazamiento de los bordes (criterio 2). La Figura 8a muestra la varianza normalizada respecto a la varianza del sistema simétrico normalizado, para cada uno de los bordes de la planta. Las líneas azules, muestran el comportamiento de los bordes de la planta cuando la estructura no está controlada, mientras que las líneas rojas muestran los desplazamientos de los bordes para el modelo $\mathrm{M}_{1}$. Por otro lado, las líneas continuas muestran el comportamiento del borde flexible (borde de la izquierda de la planta), mientras que las líneas segmentadas muestran el comportamiento del borde rígido (borde de la derecha de la planta). En la Figura 8 se observa que cuando la estructura no está controlada por los ACLS, no existe balance torsional para ninguna excentricidad. Al incluir los ACLS se produce un balance torsional perfecto, en un mayor rango de excentricidades cuando se utiliza el criterio 2, que no es el propuesto por de la Llera et al. (2005).
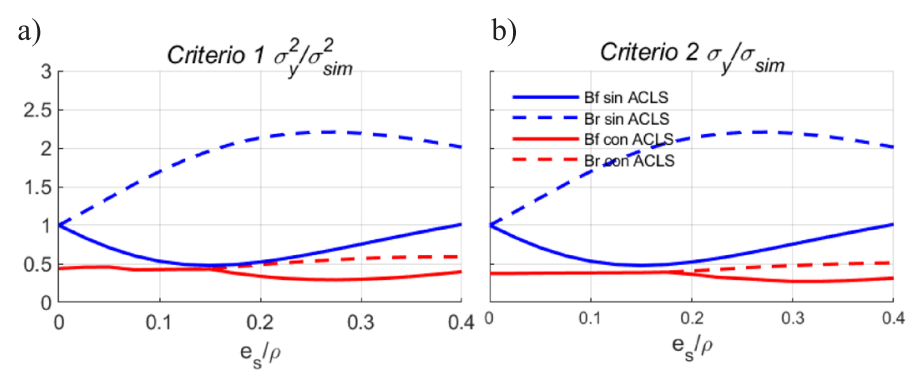

Figura 8: Posiciones óptimas, $p_{1}=p_{2}=0.5, \xi_{s}=0.02, \Omega=0.7, \mu_{l}$ $=0.06:$ a) criterio $1 \mathrm{y} \mathrm{b}$ ) criterio 2

Considerando una razón de masa mayor, $\mu_{1}=0.08$, los resultados obtenidos son idénticos para los dos criterios considerados, cuando se realizan los mismos análisis de la Figura 8. La Figura 9 muestra los resultados de la verificación del balance torsional, para una estructura de $\Omega=1.3$ y razón de masa $\mu_{1}=0.06$. La distribución de los resultados y parámetros de análisis es análogo a los presentados en la Figura 8. Se observa que el comportamiento del balance torsional es independiente del tipo de criterio utilizado. Por otra parte, los gráficos muestran, que si bien el balance torsional es perfecto, se verifica en un rango de excentricidades menor a una estructura torsionalmente flexible, para excentricidades mayores se produce un balance torsional débil, de un orden no alejado al balance perfecto. 
a)

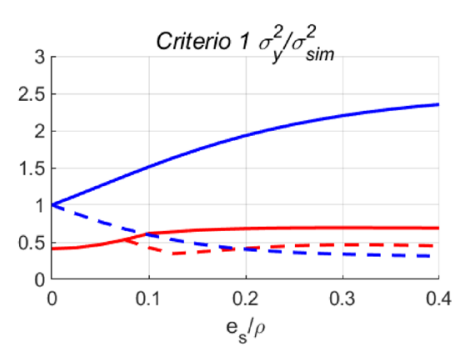

Figura 9: Posiciones óptimas, $p_{1}=p_{2}=0.5, \xi_{s}=0.02, \Omega=1.3, \mu_{l}$ $=0.06$ : a) criterio $1 \mathrm{y} \mathrm{b}$ ) criterio 2

La Figura 10, al igual que en el caso anterior, considera una estructura con una razón de frecuencia torsional igual a 1.3 y una razón de masa mayor $\mu_{1}=0.08$. Se observa que los resultados son insensibles al criterio de optimización considerado. Por otra parte, de las Figuras 9 y 10 se observa que cuando la razón de masa es mayor, el rango de excentricidades para el cual BT es perfecto (cuando los bordes alcanzan el criterio 1 ó 2 en la misma magnitud, coincidiendo la línea roja continua y la segmentada), es mayor. En el caso particular de la Figura 10, se alcanza un BT perfecto para todo el rango de excentricidades considerado en el estudio.

a)
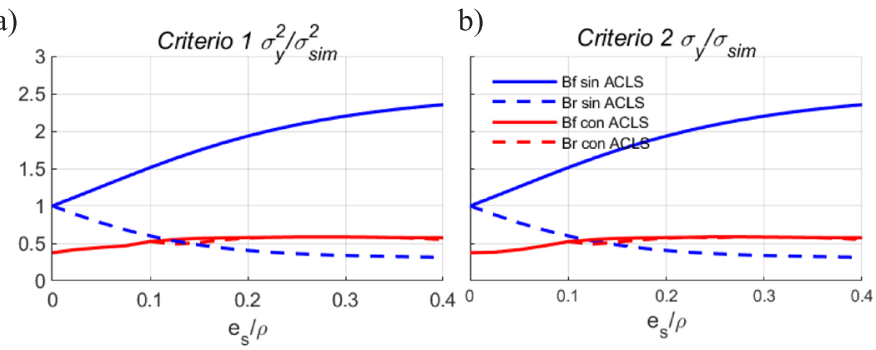

Figura 10: Posiciones óptimas, $p_{1}=p_{2}=0.5$, $\xi_{s}=0.02, \Omega=1.3$, $\mu_{l}=0.08$ : a) criterio $1 \mathrm{y} \mathrm{b}$ ) criterio 2

\section{Conclusiones}

En este trabajo se estudió un modelo formado por una estructura monosimétrica, controlada por 2 ACLS, y sometida a una excitación sísmica BBP. A partir de los resultados obtenidos se puede concluir lo siguiente:

El comportamiento de los parámetros óptimos de razón de frecuencia y posición en planta de los ACLS depende de la razón de frecuencia torsional de la estructura.

En estructuras torsionalmente flexibles, las razones de frecuencias y posiciones óptimas de los ACLS son sensibles al criterio de optimización considerado.
El coeficiente de pérdida de carga, independientemente de la razón de frecuencia torsional de la estructura, es insensible al criterio de optimización considerado.

La posición óptima de los ACLS para excentricidades mayores a 0.12 sigue la misma regla de comportamiento de los AMS (Almazán et al., 2012)

En general, para los dos criterios de optimización estudiados se obtiene el mismo comportamiento de balance torsional en la estructura, a excepción del caso de una razón de frecuencia torsional de 0.7 y una razón de masa menor.

\section{Agradecimientos}

Los autores agradecen a la Universidad del Bío-Bío, por el apoyo dado a través del Proyecto Regular UBB 2060542 IF/R.

\section{Referencias}

Almazán, J.L. and de la Llera, J.C. (2009). Torsional balance as new design criterion for asymmetric structures with energy dissipation devices. Earthquake Engineering and Structural Dynamics 38(12), 1421-1440

Almazán, J.L., Espinoza, G. and Aguirre, J.J. (2012). Torsional balance of asymmetric structures by means of tuned mass dampers. Engineering Structures 42, 308-328

Chakraborty, S., Debbarma, R. and Marano, G.C. (2012). Performance of tuned liquid column dampers considering maximum liquid motion in seismic vibration control of structures. Journal of Sound and Vibration 331(7), 1519-1531

Clough, R.W. and Penzien, J. (1975). Dynamics of structures. $2^{\text {nd }}$ edition, McGraw-Hill, USA

de la Llera, J.C., Almazán, J.L. and Vial, I.J. (2005). Torsional balance of plan $\square$ asymmetric structures with frictional dampers: analytical results. Earthquake Engineering \& Structural Dynamics 34(9), 1089-1108

Espinoza, G., Carrillo, C. and Suazo, A. (2018a). Analysis of a tuned liquid column damper in non-linear structures subjected to seismic excitations. Latin American Journal of Solids and Structures 15(7), e91

Espinoza, G., Neira, D. y Cifuentes, A. (2018b). Análisis de un amortiguador de masa sintonizado óptimo en estructuras asimétricas no lineales. Obras y Proyectos 23, 39-54 
Espinoza, G., Almazán, J.L., Jara, C. and Benedetti, F. (2019). Torsional balance of nonlinear asymmetrical structures by means of a tuned mass damper. Structural Control and Health Monitoring 26(11), e2442

García, M., de la Llera, J.C., Almazán, J.L. (2007). Torsional balance of asymmetric structures with viscoelastic dampers. Engineering Structures 29(6): 914-932

NCh2745 (2013). Análisis y diseño de edificios con aislación sísmica. Instituto Nacional de Normalización INN, Santiago, Chile

Roberts, J.B. and Spanos, P.D. (2003). Random vibration and statistical linearization. Dover Publications, New York, USA

Rozas, L., Boroschek, R.L., Tamburrino, A. and Rojas, M. (2016). A bidirectional tuned liquid column damper for reducing the seismic response of buildings. Structural Control and Health Monitoring 23(4), 621-640

Saitua, F., Lopez-Garcia, D. and Taflanidis, A.A. (2018). Optimization of height-wise damper distributions considering practical design issues. Engineering Structures 173,768-786

Sakai, F., Takaeda, S. and Tamaki, T. (1989). Tuned liquid column damper - New type device for suppression of building vibrations. First International Conference on High-Rise Buildings, Nanjing, China, 926-931
Seguín, C.E., Almazán, J.L. and de la Llera, J.C. (2013). Torsional balance of seismically isolated asymmetric structures. Engineering Structures 46, 703-717

Sgobba, S. and Marano, G.C. (2010). Optimum design of linear tuned mass dampers for structures with nonlinear behaviour. Mechanical Systems and Signal Processing 26(6), 1739-1755

Shum, K.M. (2009). Closed form optimal solution of a tuned liquid column damper for suppressing harmonic vibration of structures. Engineering Structures 31(1), 84-92

Shum, K.M. and Xu, Y.L. (2002). Multiple-tuned liquid column dampers for torsional vibration control of structures: experimental investigation. Earthquake Engineering and Structural Dynamics 31(4), 977-991

Won, A.Y., Pires, J.A. and Haroun, M.A. (1996). Stochastic seismic performance evaluation of tuned liquid column dampers. Earthquake Engineering and Structural Dynamics 25(11), 12591274

Yalla, S.K. and Kareem, A. (2000). Optimum absorber parameters for tuned liquid column dampers. Journal of Structural Engineering 126(8), 906-915 